\title{
Depiction of the Superior Petrosal Vein Complex by 3D Contrast-Enhanced MR Angiography
}

\author{
(D) B. Bender, (D)T.-K. Hauser, (D)A. Korn, (D). Klose, (D) M. Tatagiba, (D) E. Ernemann, and (DF.H. Ebner
}

\begin{abstract}
BACKGROUND AND PURPOSE: Intraoperative obliteration of the superior petrosal vein complex has a relevant risk of postoperative complications. A large venous diameter and the absence of anastomoses have been previously suggested as possible risk factors. 3D contrast-enhanced MRA was evaluated for the identification of superior petrosal vein anatomy.
\end{abstract}

MATERIALS AND METHODS: Twenty-five patients (10 men; age, 20-77 years) with a 3D-MRA (voxel size, $0.4 \times 0.4 \times 0.5 \mathrm{~mm}^{3}$ ) at 3T, including the posterior fossa, were retrospectively identified. Image evaluation was performed independently by 2 neuroradiologists with respect to overall image quality and the presence, location, size, tributaries, and anastomotic veins of the superior petrosal vein complex. Additionally, 8 neurosurgical cases with intraoperative validation of the venous anatomy were examined.

RESULTS: All studies were of diagnostic image quality. Interobserver agreement was excellent for image-quality measurements $(r=$ $0.751-0.982)$ and good for measured vessel size $(r=0.563-0.828)$. A total of 83 superior petrosal veins were identified. The distribution of drainage locations and identification of tributaries and anastomotic veins were consistent with previous anatomic studies. The results showed that $4.8 \%$ of superior petrosal veins had a diameter of $>2 \mathrm{~mm}$ and lacked a visible anastomosis. All surgical cases showed excellent agreement between the MRA and the intraoperative observations.

CONCLUSIONS: 3D-MRA with high resolution is appropriate for analyzing the size, course, tributaries, and anastomoses of the superior petrosal vein. A total of $4.8 \%$ of the identified superior petrosal veins had to be classified as potential high-risk veins. The measurements correlated with the intraoperative findings.

ABBREVIATIONS: SPS = superior petrosal sinus; SPV = superior petrosal vein

n the 1960s, radiologists first described the detailed anatomy of the venous drainage of the posterior fossa using conventional angiography ${ }^{1-3}$ and identified the diagnostic significance of the superior petrosal vein (SPV) in the evaluation of cerebellopontine angle tumors. ${ }^{2}$ Several neurosurgical reports have described com-

Received May 28, 2018; accepted after revision September 10.

From the Department of Radiology (B.B., T.-K.H., A.K., U.K., U.E.), Diagnostic and Interventional Neuroradiology, and Department of Neurosurgery (M.T., F.H.E.), University Hospital Tübingen, Tübingen, Germany; and Radiologie in Hof (A.K.), Hof, Germany.

Other patient samples with slightly different MR imaging protocols or small subgroups of the present article have been presented at the following meetings: Annual Meeting of the German Society of Skull Base Surgery, October 11-12, 2013; Tübingen, Germany; Plenary Annual Meeting of the European Skull Base Society, June 26-28, 2014; Paris, France; and Plenary Annual Meeting of the German Society of Neuroradiology, October 23-25, 2014; Cologne, Germany.

Please address correspondence to Benjamin Bender, MD, Department of Diagnostic and Interventional Neuroradiology, University Hospital Tübingen, Hoppe-Seyler-Str 3, 72076 Tübingen, Germany; e-mail:

benjamin.bender@med.uni-tuebingen.de

三 Indicates article with supplemental on-line table.

http://dx.doi.org/10.3174/ajnr.A5864 plications arising from the sacrifice of the SPV due to compromise of the cerebellar deep venous outflow, leading to extensive venous infarction and hemorrhage. ${ }^{4-8}$ The exact anatomic location of the SPV plays a crucial role in evaluating the risks of a middle fossa approach with anterior petrosectomy and the retrosigmoid-suprameatal approach because the intraoperative location of at-risk veins differs greatly between the 2 approaches. ${ }^{9}$ Precise knowledge of the course, size, variations, and outflow system of associated anastomotic veins of the SPV is of great interest to neurosurgeons to plan the approach and thus reduce the risk of venous complications.

The SPV enters the superior petrosal sinus (SPS) and may be formed by the terminal segment of a single vein or by the common stem formed by the union of up to 5 veins. ${ }^{6}$ The most common tributaries of the SPV are the transverse pontine and pontotrigeminal veins (depending on the literature, also considered to be part of the lateral mesencephalic vein or referred to as the brachial tributary of the superior petrosal vein), the common stem of the superior cerebellar hemispheric veins, and the veins of the cer- 
ebellopontine fissure and the middle cerebellar peduncle. ${ }^{10-12}$ The SPV can be classified as a lateral, intermediate, or medial type based on the anatomic relationships between the site of entry into the superior petrosal sinus and the internal acoustic meatus ${ }^{12}$ and based on the relationship of the site of entry into the superior petrosal sinus to the internal acoustic meatus and the Meckel cave. ${ }^{9}$ The anterior pontomesencephalic and the lateral mesencephalic veins link the SPV complex and the basal vein of Rosenthal and thus represent the 2 main anastomoses between the supra- and infratentorial venous systems. ${ }^{13,14}$ In the case of several SPVs, there is also the possibility of an anastomosis between them.

Many MR imaging studies have shown that venous structures can be depicted with high diagnostic accuracy. ${ }^{15-20}$ However, due to the variable diameter, the close relationship to nearby air-filled bone structures and the superior cerebellar artery, and the course along brain parenchyma, CSF, and bone, the depiction of the SPV and its tributaries is challenging. Knowledge about the size, tributaries, and anastomoses of the SPV mostly exists from microsurgical and anatomic studies ${ }^{9-12,14,21}$ or digital subtraction angiography. ${ }^{11}$ A detailed preoperative MR imaging evaluation of the individual venous anatomy would meaningfully inform the surgical strategy.

The purpose of our study was to evaluate whether a contrastenhanced MRA with high spatial resolution that cannot be obtained with $2 \mathrm{D}$ noncontrast venography can depict the variations, tributaries, and anastomoses of the SPV.

\section{MATERIALS AND METHODS \\ Patients}

All patients who underwent a high-resolution 3D-MRA at the 3T MR imaging scanner of the Department of Radiology, University Hospital Tübingen in 2013 were retrospectively identified by a radiologic information system search; patients younger than 18 years of age were excluded. A total of 25 patients were identified (10 men, 15 women; mean age, $49.3 \pm 19.5$ years; age range, 20-77 years) and included in the analysis. After transferring the clinical data and the results of the image evaluation into a research data base, we removed all identifying information before further evaluation. There was a broad spectrum of clinical indications.

Additionally, 8 patients with cerebellopontine angle tumors underwent preoperative 3D-MRA. For these cases, a comparison with the intraoperative findings from the surgical report and video documentation was conducted. Our study was approved by the local institutional review board, and the need for informed consent was waived due to the anonymous evaluation.

\section{Imaging Technique}

All studies were performed on a 3T MR imaging system (Magnetom Skyra; Siemens, Erlangen, Germany) with a 24-channel headneck coil.

The 3D-MRA was performed 20 seconds after an intravenous injection of Gd-DTPA $(0.1 \mathrm{mmol} / \mathrm{kg}$ body weight $)$ at $1.5 \mathrm{~mL} / \mathrm{s}$, followed by a saline flush $(40 \mathrm{~mL})$ at $1.5 \mathrm{~mL} / \mathrm{s}$ using an electronic power injector (Spectris MR injector; MedRad, Indianola, Pennsylvania). A fast $3 \mathrm{D}$ gradient recalled-echo sequence in the axial orientation was used (FOV, $240 \times 210 \mathrm{~mm}^{2}$; matrix, $576 \times 504 ; 1$ slab; 176 slices; slice thickness, $0.5 \mathrm{~mm}$; TR/TE, 8.44/2.8 ms; flip angle, $30^{\circ}$; bandwidth, $130 \mathrm{~Hz} /$ pixel), with elliptic centric $k$-space encoding, asymmetric $k$-space sampling (partial Fourier 6/8), and zero interpolation in all 3 planes.

Subsequent image-quality analysis was performed independently by 2 neuroradiologists, each with 6 years of experience. For cases in which the reported number of SPVs differed, a third senior neuroradiologist with 16 years of experience evaluated the case in a consensus reading with the first 2 raters. The original image data and thin-slab MIPs (3-mm-thick, reconstructed in steps of $1 \mathrm{~mm}$ ) aligned to the superior petrosal sinus in all 3 axes were evaluated on the local PACS and a 3D volume viewer (syngo.via Siemens Healthineers, Erlangen, Germany). Separate image-reading sessions were organized for both readers. The MIP reconstructions were used to obtain a first overview of the relationship among the different tributaries of the SPVs. In all cases, an overlap between adjacent vessels was seen and the original image data were needed to separate branch communications from artificial overlap.

The readers were asked to assess the overall quality of MR angiograms with regard to venous enhancement and the presence of artifacts and/or noise using a 1-3 scoring scale (1, poor image quality: inadequate venous enhancement and/or the presence of relevant artifacts/noise impairing the diagnosis; 2, good image quality, sufficient for diagnosis: adequate venous enhancement and/or mild-to-moderate artifacts/noise, not interfering with diagnosis; and 3, excellent image quality for a highly confident diagnosis: good venous enhancement, no or minimal artifacts/noise).

Both raters were asked to draw an oval ROI in the basilar artery, brain stem, outside the brain within the noise, in the left and right transversal sinuses, and in the left and right petrosal sinuses. Within this ROI, mean signal intensity and SD were recorded.

\section{Anatomic Analysis}

According to the anatomic definition, every vein draining into the SPS was classified as an SPV. For the evaluation of the tributaries, the rater used a previously reported scheme that divides the tributaries into 4 groups: the anterior pontomesencephalic group, the tentorial group, the petrosal group, and the posterior mesencephalic group (Fig 1). ${ }^{11}$ Each rater evaluated the presence and sizes of anastomoses between the supra- and infratentorial venous systems via the anterior pontomesencephalic and the posterior mesencephalic groups. If there was no direct anastomosis of an SPV, the raters were asked to judge whether an anastomosis to another SPV with infra- to supratentorial anastomosis was present. If 2 anastomoses (with diameter $d_{1}$ and $d_{2}$ ) were present for an SPV, the combined diameter $d_{c}$ was calculated as follows:

$$
d_{c}=2 \times \sqrt{\left(\frac{d_{1}}{2}\right)^{2} \pi+\left(\frac{d_{2}}{2}\right)^{2} \pi} .
$$

The SPV drainages were subdivided into lateral, intermediate, and medial groups based on the relationship between the site of entry into the superior petrosal sinus and the internal acoustic meatus on sagittal MPR reconstructions: Type I was defined as an 


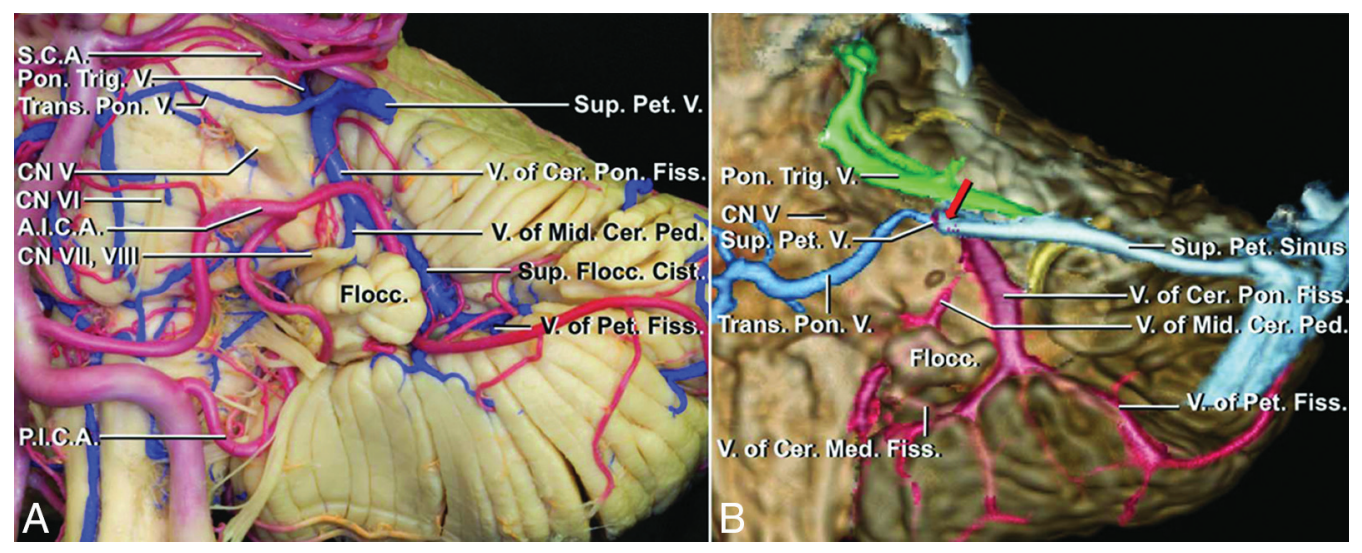

FIG 1. Anatomic overview of the tributaries of the superior petrosal veins (reproduced with permission of Oxford University Press from Matsushima K, Matsushima T, Kuga Y, et al. Classification of the superior petrosal veins and sinus based on drainage pattern. Neurosurgery 2014;10 Suppl 2:357-67)." A, Anterior view of the left petrosal cerebellar surface and anterolateral brain stem of an anatomic preparation with veins perfused with blue and arteries with red silicone. $B$, Colored multifusion image (T1 MPRAGE and conventional 3D digital subtraction angiography) with a similar view compared with $A$. The 4 drainage groups are red (petrosal group), blue (anterior pontomesencephalic group), green (posterior mesencephalic group), and yellow (tentorial group).

SPV that drains into the SPS lateral to the internal acoustic meatus, SPV type II drains into the SPS above the internal acoustic meatus, and type III drains into the SPS medial to the internal acoustic meatus. ${ }^{12}$

The sizes of the basal vein, SPV, anterior pontomesencephalic vein, and lateral mesencephalic vein were measured in millimeters. If the size was $<0.5 \mathrm{~mm}$ (and therefore smaller than the acquired resolution), a value of $0.2 \mathrm{~mm}$ was used for statistical evaluation.

\section{Statistical Evaluation}

All statistical analyses were performed with SPSS (Version 22; IBM, Armonk, New York). Image-quality scores were plotted as median and range. A Wilcoxon rank sum test was used to evaluate the significance of the image-quality grading differences between the 2 readers. A Kruskal-Wallis test was used for between-group differences; post hoc analysis with correction for multiple comparisons was performed using the Dunn-Bonferroni test. To evaluate the effect size of the between-group differences, we calculated Pearson correlation coefficients $(r)$ for significant results $(0.1=$ small, $0.3=$ medium, $0.5=$ large effect ${ }^{22}$ ).

Interobserver agreement for subjective image-quality grading was determined by calculating the linear weighted $\kappa$ coefficient (poor agreement, $\kappa=0$; slight agreement, $\kappa=0.01-0.2$; fair agreement, $\kappa=0.21-0.4$; moderate agreement, $\kappa=0.41-0.6$; good agreement, $\kappa=0.61-0.8$; and excellent agreement, $\kappa=$ $\left.0.81-1^{23}\right)$. Interobserver agreement for objective image-quality measurements was evaluated by correlation coefficient analysis.

A 2-sided value of $P<.05$ was considered significant.

\section{RESULTS}

We examined 50 SPSs in 25 patients. Image quality was assessed as excellent in 13/25 cases by the first reader (B.B.) and in 17/25 cases by the second reader (A.K.); all other cases were considered good quality. The interobserver reliability was good $(\kappa=0.675)$. Figure 2 shows a typical MIP reconstruction at the level of the inflow of the SPV into the SPS.

Between raters, there was excellent correlation in the mea-

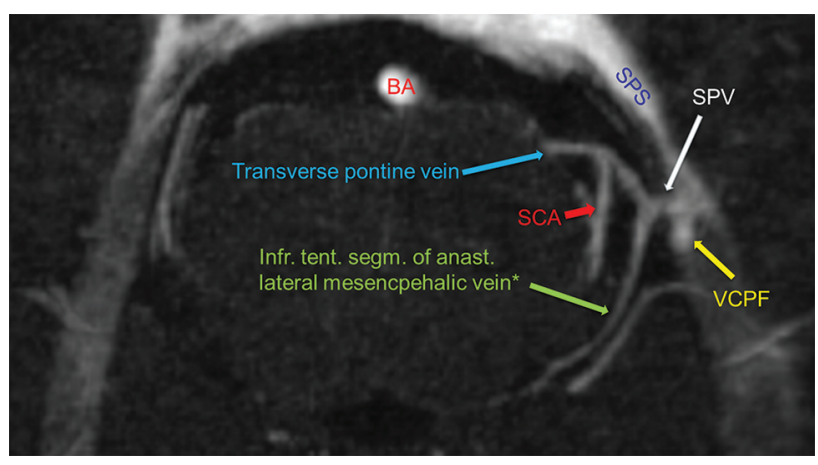

FIG 2. Transversal MIP at the level of the drainage of the SPV into the SPS (type II, internal acoustic meatus not shown). The tributary veins are clearly identifiable: lateral mesencephalic vein (asterisk, posterior mesencephalic group, anastomosis to the basal vein), transverse pontine vein (anterior pontomesencephalic group), and vein of cerebellopontine fissure (petrosal group). The artificial union of the SCA and the transverse pontine vein is clearly distinguishable on the original imaging data (asterisk, depending on the literature, also named pontotrigeminal vein and brachial tributary of the superior petrosal vein). BA indicates basilar artery; SCA, superior cerebellar artery; VCPF, vein of cerebellopontine fissure; infr. tent. segm. of anast, infratentorial segment of anastomosing.

sured signal intensities for the anatomic ROIs (Table 1) and good agreement for the measured diameters (Table 2). Objective image criteria of the area of interest were excellent; the mean SNR of the left SPS was 141.6 (range, 72.2-300.4), and the mean SNR of the right SPS was 143.2 (range, 76.5-273.6).

The first and second raters identified 42 and 48 SPVs, respectively, on the left side and 37 and 44 SPVs on the right side. The interrater reliability was moderate for both the right $(\kappa=0.496)$ and left $(\kappa=0.502)$ hemispheres.

Ten and 7 cases with a divergent judgment by the 2 reviewers on the left and right sides, respectively, were evaluated a second time in a consensus reading. After the consensus readings, 43 SPVs on the left side and 40 SPVs on the right side were identified. In all except 2 cases with a divergent judgment, the point of drainage of a transverse pontine vein (type III) was considered to be the SPS by one rater and the cavernous sinus by the other rater. In these cases, a line was drawn through the unambigu- 
Table 1: Results of the ROI analysis for objective image quality and the calculated correlation coefficients for interobserver agreement ${ }^{a}$

\begin{tabular}{|c|c|c|c|c|c|}
\hline Anatomic Structure & Mean Rater 1 (A.U.) & Mean Rater 2 (A.U.) & No. & $\begin{array}{l}\text { Pearson Correlation } \\
\text { Coefficient } R\end{array}$ & Significance \\
\hline Noise $^{a}$ & $4 \pm 1$ & $4 \pm 1$ & 25 & 0.818 & $<.001$ \\
\hline Basilar artery & $1110 \pm 294$ & $1170 \pm 381$ & 25 & 0.852 & $<.001$ \\
\hline Brain stem & $176 \pm 24$ & $179 \pm 24$ & 25 & 0.895 & $<.001$ \\
\hline Vein of Galen & $1040 \pm 344$ & $1020 \pm 303$ & 23 & 0.910 & $<.001$ \\
\hline Transverse sinus (R) & $905 \pm 280$ & $896 \pm 276$ & 25 & 0.982 & $<.001$ \\
\hline Transverse sinus (L) & $878 \pm 232$ & $902 \pm 244$ & 25 & 0.982 & $<.001$ \\
\hline Basal vein $(R)$ & $812 \pm 283$ & $783 \pm 243$ & 19 & 0.910 & $<.001$ \\
\hline Basal vein (L) & $847 \pm 233$ & $839 \pm 234$ & 24 & 0.936 & $<.001$ \\
\hline SPS (R) & $561 \pm 186$ & $601 \pm 183$ & 25 & 0.852 & $<.001$ \\
\hline SPS (L) & $537 \pm 233$ & $612 \pm 169$ & 25 & 0.741 & $<.001$ \\
\hline
\end{tabular}

Note:-R indicates right; L, left; A.U., arbitrary units; No., number of cases.

${ }^{a}$ For noise, the SD of the signal in the ROI is given; for all other ROIs, the mean signal is presented.

Table 2: Results of the evaluation of the diameters of the main vessels ${ }^{a}$

\begin{tabular}{|c|c|c|c|c|c|}
\hline Anatomic Structure & Mean Rater 1 (A.U.) & Mean Rater 2 (A.U.) & No. & $\begin{array}{l}\text { arson Correlatic } \\
\text { Coefficient } R\end{array}$ & Significance \\
\hline Basal vein (R) & $1.7 \pm 0.4$ & $1.6 \pm 0.8$ & 24 & 0.563 & .003 \\
\hline Basal vein (L) & $1.8 \pm 0.4$ & $1.7 \pm 0.4$ & 25 & 0.659 & $<.001$ \\
\hline Lateral mesencephalic vein (R) & $0.9 \pm 0.5$ & $0.9 \pm 0.5$ & 19 & 0.753 & $<.001$ \\
\hline Lateral mesencephalic vein (L) & $0.7 \pm 0.7$ & $0.8 \pm 0.6$ & 20 & 0.828 & $<.001$ \\
\hline Anterior pontomesencephalic vein & $0.6 \pm 0.6$ & $0.6 \pm 0.5$ & 25 & 0.726 & $<.001$ \\
\hline SPS (R) & $2.6 \pm 0.5$ & $2.5 \pm 0.7$ & 25 & 0.663 & $<.001$ \\
\hline SPS (L) & $2.6 \pm 0.6$ & $2.5 \pm 0.6$ & 25 & 0.595 & .002 \\
\hline
\end{tabular}

Note:-No. indicates the number of veins present; R, right; L, left; A.U., arbitrary units.

${ }^{a}$ Missing veins were omitted for the calculation of the mean and rated as $0 \mathrm{~mm}$ for the correlation coefficient. Visible veins $<0.5 \mathrm{~mm}$ were rated as $0.2 \mathrm{~mm}$.

Table 3: Position and size of 83 petrosal veins in 25 patients without intraoperative validation and in 8 patients with intraoperative validation

\begin{tabular}{lccccc}
\hline & \multicolumn{2}{c}{ No. of SPVs in Patients } & & \multicolumn{2}{c}{ Mean Diameter (Range) (mm) in Patients } \\
\cline { 2 - 3 } \cline { 5 - 6 } Type of SPV & Without Surgery & With Surgery & & Without Surgery & With Surgery \\
\hline I, lateral & $13(15.7 \%)$ & $1(4.8 \%)$ & $2.0(0.5-2.9)$ & $2.5(2.5-2.5)$ \\
II, intermediate & $41(49.4 \%)$ & $10(47.6 \%)$ & & $2.4(<0.5-5.6)$ & $2.1(1.5-3.6)$ \\
III, medial & $29(34.9 \%)$ & $10(47.6 \%)$ & & $1.3(<0.5-2.7)$ & $1.0(<0.5-2.1)$ \\
I-III & 83 & 21 & $2.0(<0.5-5.6)$ & $1.6(<0.5-3.6)$ \\
\hline
\end{tabular}

Table 4: Frequencies of superior petrosal vein drainage groups for each cerebellopontine angle $(n=50)$ and each superior petrosal vein $(n=83)$

\begin{tabular}{|c|c|c|c|c|c|c|}
\hline \multirow[b]{2}{*}{ Drainage Group } & \multicolumn{3}{|c|}{ Frequency of Group/Side } & \multicolumn{3}{|c|}{ Frequency of Group/SPV } \\
\hline & Left & Right & Total & Left & Right & Total \\
\hline Petrosal & 25 & 24 & $49 / 50$ sides ( $98 \%)$ & 31 & 31 & $62 / 83(74.7 \%)$ \\
\hline Posterior mesencephalic & 22 & 22 & $44 / 50$ sides ( $88 \%)$ & 22 & 23 & $45 / 83(54.2 \%)$ \\
\hline Anterior pontomesencephalic & 23 & 21 & $44 / 50$ sides ( $88 \%)$ & 27 & 27 & $54 / 83(65.1 \%)$ \\
\hline Tentorial & 14 & 15 & $29 / 50$ sides $(58 \%)$ & 14 & 15 & $29 / 83(34.9 \%)$ \\
\hline
\end{tabular}

ous course of the SPS; if a medial vein drained at the level of this line, it was considered an SPV during the consensus reading. If the drainage was medial to or below this line, it was not considered an SPV.

After the consensus readings, 22/50 SPSs (44\%) had 1 SPV, 23/50 SPSs (46\%) had 2 SPVs, and 5/50 (10\%) had 3 SPVs. Table 3 summarizes the types and diameters of the SPVs. There was a significant difference in vein size for the 3 types $(\mathrm{H}(2)=28,871$, $P<.001)$. Post hoc comparison indicated that type III veins were significantly smaller than type I $(z=2.861, P=.01, r=0.44)$ and type II ( $z=5.333, P<.001, r=0.64)$ veins. Table 4 shows the distribution of the drainage groups. The most common tributary vein was the vein of the cerebellopontine fissure (petrosal group), which drained into an SPV in all except 1 case. Eighteen of the 83 SPVs $(21.7 \%)$ had no visible infra-to-supratentorial anastomosis and were considered potentially dangerous SPVs in case of oblit- eration. Of these 18 SPVs, 2 were type I (2.4\% of all SPVs; $15.4 \%$ of type I), 6 were type II (7.2\% of all SPVs; $14.6 \%$ of type II), and 10 were type III ( $12 \%$ of all SPVs; $34.5 \%$ of type III). The mean diameter of the potentially dangerous SPVs was $1.5 \mathrm{~mm}(<0.5-3$ $\mathrm{mm}$ ). Figure 3 illustrates the diameter of the SPVs in comparison with the diameter of the anastomosis.

Data for the intraoperative validation group are shown in Table 3 and On-line Table 1. In general, the distribution and size were comparable with those in the patient group without intraoperative validation. Many SPVs on the affected side were compressed at the entry into the SPS, with enlarged anastomoses. In all cases, there was excellent agreement with the intraoperative anatomy. In 2 cases, an SPV was sacrificed during an operation without adverse effects (patient 3, Fig 4 and patient 6, second type II SPV). In patient 1, the left SPV was preserved, though altered arachnoid mater and tumor covering parts of the SPV 


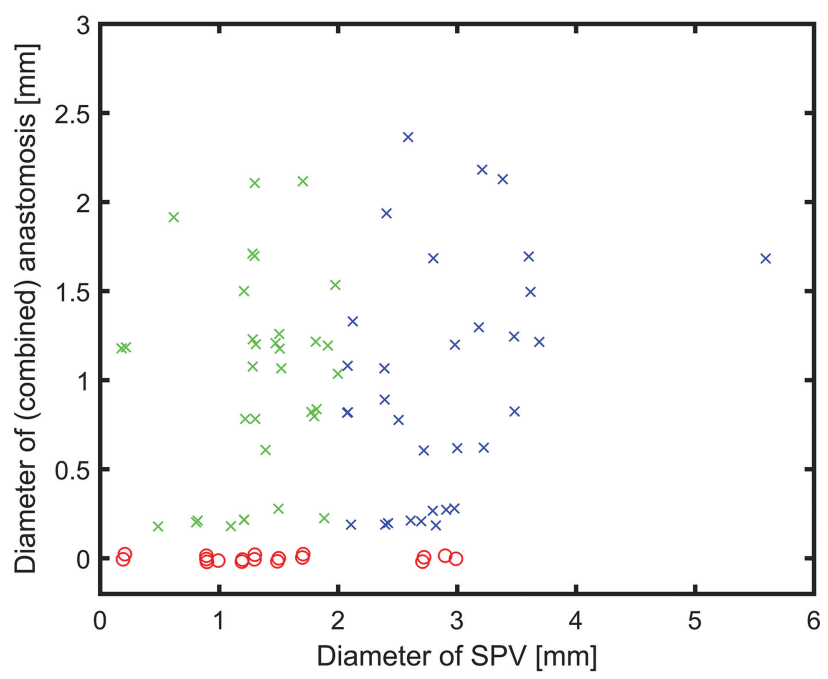

FIG 3. Scatterplot of the diameter of the SPV versus the combined diameters of its anastomoses. To better visualize overlaying data points, we added a random offset between -0.025 and 0.025 to each diameter. SPVs with no visible anastomosis are shown as red circles, while $x$ represents SPVs with at least 1 anastomosis (green indicates the diameter of the SPV $\leq 2 \mathrm{~mm}$; blue, diameter of the SPV of $>2 \mathrm{~mm}$ ). SPVs with a diameter of $>2 \mathrm{~mm}$ are considered dangerous by Zhong et al. ${ }^{6}$ were seen (Fig 5). Because the fresh-frozen section was not conclusive, a radical approach with a sacrifice of the SPV was considered too dangerous, given the information from the preoperative MR imaging.

\section{DISCUSSION}

In this study, 3D-MRAs were retrospectively evaluated for image quality and anatomic depiction of the SPV. Objective imaging criteria and interrater reliability regarding subjective image quality were good to excellent. Anatomic depiction of the SPV and identification of its tributaries and anastomoses were possible in all cases, and the results are in good agreement with previous results. ${ }^{9-12}$ There is a large variation in the reported frequency of drainage types. ${ }^{9,11,12}$ This variation can be explained, in part, by the difficulty in defining the exact border of the internal auditory meatus, as well as a slightly different classification scheme in 1 of the studies. ${ }^{9}$

In contrast to previous studies, ${ }^{9,11}$ SPSs with 2 or 3 draining SPVs were more common, with $42 \%$ and $10 \%$, respectively, in comparison with $20 \%$ and $3 \%{ }^{11}$ and $23 \%$ and $0 \% .{ }^{9}$ However, these frequencies are consistent with an earlier microsurgical study that found frequencies of $50 \%$ and $10 \% .{ }^{12}$ Only Huang et

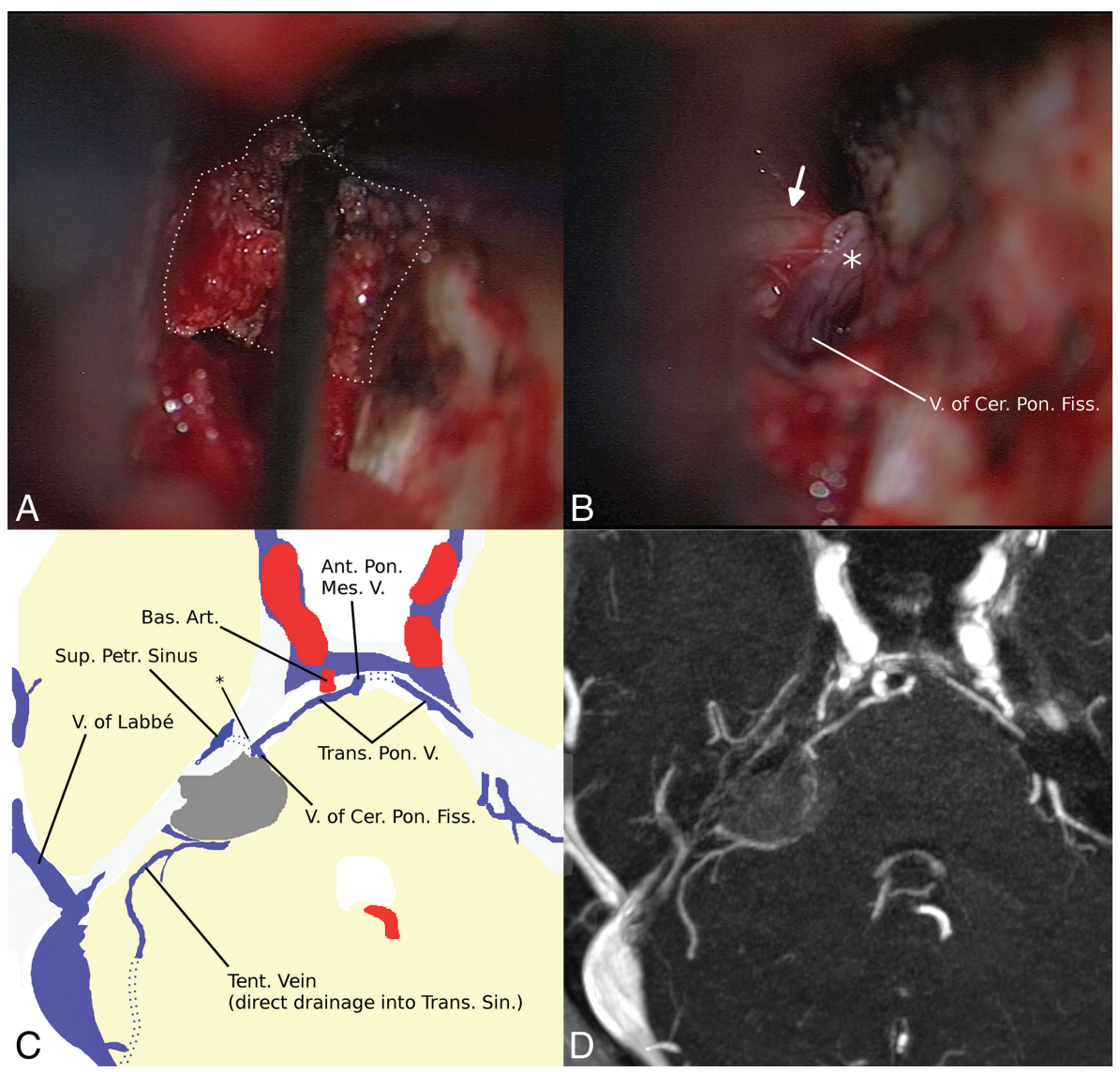

FIG 4. $A$, Intraoperative view shows that the venous anatomy is hidden for the neurosurgeon by the meningioma (white dots). B, After removal of the tumor, the neurosurgeon can see the medial superior petrosal vein (type III) and the trigeminal nerve (arrow). Two tributaries are encountered in concordance with the MR imaging. A schematic drawing $(C)$ based on axial MIP reconstruction $(D)$ of the preoperative 3D-MRA shows a compressed single SPV (asterisk in C) just ventral to the meningioma, with 2 tributaries and a main drainage via a pontine transverse vein and the anterior pontomesencephalic vein. Although the location was known, partial thermocoagulation (note the different color of the SPV marked with an asterisk in B) of the previously compressed SPV could not be prevented. The dorsal region of the cerebellum had direct drainage into the basal vein via a large lateral mesencephalic vein (not shown). V indicates vein; V. of Cer. Pon. Fiss, vein of the cerebellopontine fissure; Bas. Art., basal artery; Ant. Pon. Mes. V, anterior pontomesencephalic vein; Sup. Petr. Sinus, superior petrosal sinus; Trans. Pon. V., transverse pontine vein; Tent. Vein, tentorial vein; Trans. Sin, transverse sinus. 


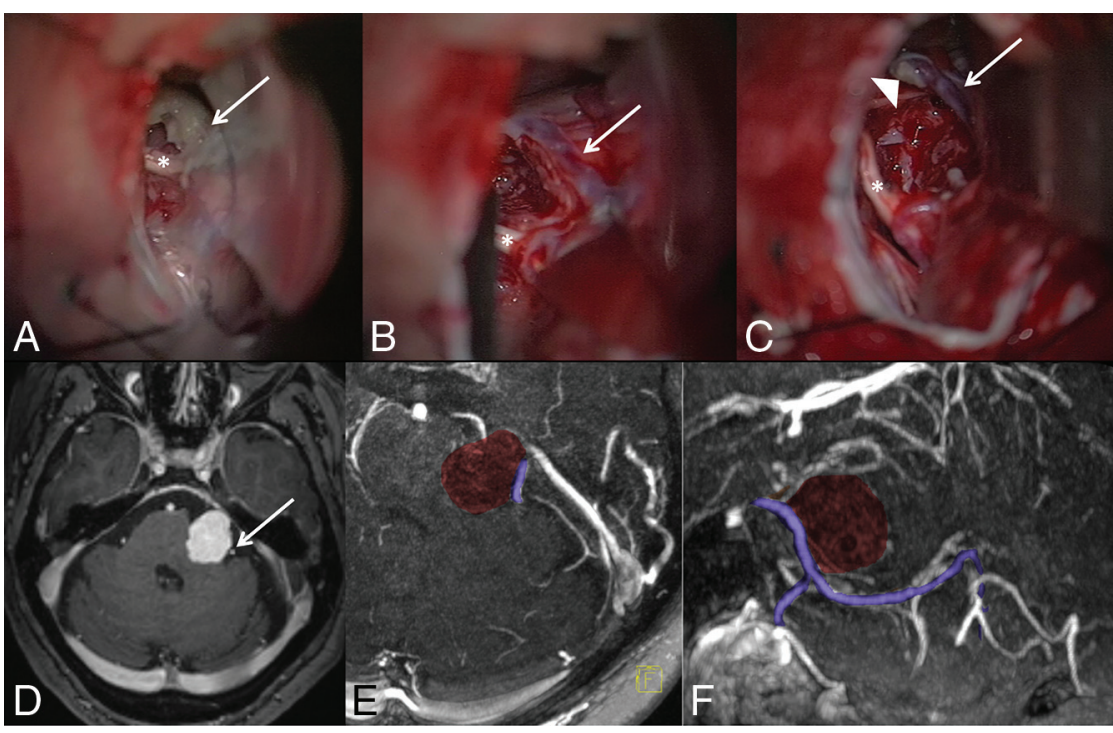

FIG 5. Intraoperative views of the tumor ( $A$, white arrow) and after removal of the tumor ( $B$ and $C)$ : TI-weighted postcontrast scan $(D)$ and axial $(E)$ and sagittal $(F)$ MIP reconstructions of the MR imaging. Nerves VII and VIII (asterisk) are seen directly below the tumor. A single large SPV (white arrow in $B-D$; purple colored in E,F) drains a large portion of the cerebellum and enters the SPS just in front of the tumor (type II). With a diameter of $>2 \mathrm{~mm}$ and no visible collaterals in the MRA and intraoperatively, fibrous arachnoidea and tumor (arrowhead) sticking to the SPV were not removed due to the high risk of obliteration.

$\mathrm{al}^{3}$ noted that "not infrequently there are several venous stems which drain into the superior petrosal sinus at different points."

Consistent with a previous study, ${ }^{11}$ we found that the petrosal group was the largest and most common drainage group of the SPV and was present in all except 1 SPS. The posterior mesencephalic and the anterior pontomesencephalic groups were equally common and were present in $88 \%$ of SPSs, in agreement with a previous study, ${ }^{11}$ considering the number of subjects evaluated in both studies. A noticeable difference was found for the tentorial group, which was found in only $58 \%$ of our patients but in $70 \%$ in the study by Matsushima et al. ${ }^{11}$ The largest tributary of the tentorial group, the anterior lateral marginal vein, ${ }^{11}$ is a very small vein and is the only vein for which the terminal size has been described as "very small" without a corresponding measurement (millimeters) in a microsurgical anatomic study. ${ }^{12} \mathrm{With}$ a resolution of $0.42 \times 0.42 \times 0.5 \mathrm{~mm}$, our MR image, therefore, might have missed some of the very small anterior lateral marginal veins with drainage into the SPS, and this group might be underestimated in our sample. Very small veins are usually not considered a problem during an operation ${ }^{6}$; therefore, the insufficient resolution for these veins is of no clinical relevance. In comparison with a recent study based on CT-venography, ${ }^{24}$ 3D-MRA depicted more tributaries, probably due to the better resolution. Both methods can be easily incorporated into the standard work-up of planned surgical cases.

In the neurosurgical literature, the rate of complications after sacrificing an SPV varies. In a study of 30 patients with dissection of an SPV during resection of a petrous apex meningioma, minor complications were described in $23.3 \%$, and major complications, in $6.6 \%$ of patients. ${ }^{4}$ Zhong et $\mathrm{al}^{6}$ identified $5 / 205$ potential high-risk SPVs (2.4\%) in patients undergoing microvascular decompression for trigeminal neuralgia. In 3/5 cases, the SPV was occluded to achieve a decompression of the root entry zone, despite deterioration of the electrophysiologic recordings. All patients developed a major complication with cerebellar edema, brain shift, and the need for posterior fossa decompression. It is unclear from the publication whether minor complications occurred or whether they were evaluated. Anastomoses do not exist between the right and left SPV complex but only between the ipsilateral infratentorial and supratentorial compartments. ${ }^{14}$ In our study, we were able to evaluate the presence and size of the 2 major anastomotic pathways (the lateral mesencephalic vein and anterior pontomesencephalic vein) of the SPV to the deep supratentorial venous system, which are considered crucial for risk stratification. ${ }^{14} \mathrm{~A}$ third pathway formed by tiny branches, the anterolateral anastomosis, was not visible in our study, probably due to its size. Due to the small diameter, the role of the anterolateral anastomosis as a sufficient alternative drainage after occlusion of a large SPV has to be discussed.

A total of $43.4 \%$ of the SPVs in our study were $>2 \mathrm{~mm}$ and must be considered dangerous following the definition of Zhong et al. ${ }^{6}$ A total of $22 \%$ of SPVs had no visible anastomoses. In total, $4 / 18$ SPVs without visible anastomoses were $>2 \mathrm{~mm}$. The frequencies of SPVs of $>2 \mathrm{~mm}$ lacking anastomoses $(4.8 \%)$ and SPVs of $\leq 2$ $\mathrm{mm}$ without visible anastomoses $(16.9 \%)$ nicely correlate with the rate of major $(2.4 \%-6.6 \%)$ and minor $(23.3 \%)$ complications in the literature. $^{4,6}$

Usually, veins are elastic and can increase in size if needed. For the pulmonary vein, a variability in the diameter of approximately $15 \%$, as the heart cycles, can be found in the literature. ${ }^{25}$ Additionally, very small anastomoses (not visible in our study) can probably further increase the flow through alternative pathways. It remains unclear how large the diameters of the visible anastomoses have to be in comparison with the SPV if it is sacrificed. In all surgical cases, the intraoperative anatomy correlated well with the findings of the preoperative MR imaging. In 1 case, a large SPV of $2.1 \mathrm{~mm}$ had to be sacrificed, and the visible anastomosis increased in size at follow-up examinations.

\section{CONCLUSIONS}

This study shows that with dedicated high-resolution MR angiography of the posterior fossa, one can identify the size, location, and presence of anastomoses of the SPV. This procedure permits the preoperative identification of patients at risk for postoperative complications if the SPV is sacrificed. Our data support the hypothesis that the combination of a largediameter SPV and the absence of sufficient anastomoses might be a risk factor for complications after intraoperative sacrifice of the SPV. 
Disclosures: Benjamin Bender-UNRELATED: Consultancy: Medtronic*; Travel/Accommodations/Meeting Expenses Unrelated to Activities Listed: Bayer Vital.* *Money paid to the institution.

\section{REFERENCES}

1. Huang YP, Wolf BS. The veins of the posterior fossa-superior or galenic draining group. Am J Roentgenol Radium Ther Nucl Med 1965;95:808-21 CrossRef Medline

2. Takahashi M, Wilson G, Hanafee W. The significance of the petrosal vein in the diagnosis of cerebellopontine angle tumors. Radiology 1967;89:834-40 CrossRef Medline

3. Huang YP, Wolf BS, Antin SP, et al. The veins of the posterior fossaanterior or petrosal draining group. Am J Roentgenol Radium Ther Nucl Med 1968;104:36-56 CrossRef Medline

4. Koerbel A, Gharabaghi A, Safavi-Abbasi S, et al. Venous complications following petrosal vein sectioning in surgery of petrous apex meningiomas. Eur J Surg Oncol 2009;35:773-79 CrossRef Medline

5. Koerbel A, Wolf SA, Kiss A. Peduncular hallucinosis after sacrifice of veins of the petrosal venous complex for trigeminal neuralgia. Acta Neurochir(Wien) 2007;149:831-32; discussion 832-33 CrossRef Medline

6. Zhong J, Li ST, Xu SQ, et al. Management of petrosal veins during microvascular decompression for trigeminal neuralgia. Neurol Res 2008;30:697-700 CrossRef Medline

7. Masuoka J, Matsushima T, Hikita T, et al. Cerebellar swelling after sacrifice of the superior petrosal vein during microvascular decompression for trigeminal neuralgia. J Clin Neurosci 2009;16:1342-44 CrossRef Medline

8. Inamasu J, Shiobara R, Kawase T, et al. Haemorrhagic venous infarction following the posterior petrosal approach for acoustic neurinoma surgery: a report of two cases. Eur Arch Otorhinolaryngol 2002; 259:162-65 CrossRef Medline

9. Tanriover N, Abe H, Rhoton AL Jr, et al. Microsurgical anatomy of the superior petrosal venous complex: new classifications and implications for subtemporal transtentorial and retrosigmoid suprameatal approaches. J Neurosurg 2007;106:1041-50 CrossRef Medline

10. Rhoton AL Jr. The posterior fossa veins. Neurosurgery 2000;47: S69-92 Medline

11. Matsushima K, Matsushima T, Kuga Y, et al. Classification of the superior petrosal veins and sinus based on drainage pattern. $\mathrm{Neu}$ rosurgery 2014;10(Suppl 2):357-67; discussion 367 CrossRef Medline

12. Matsushima T, Rhoton AL Jr, de Oliveira E, et al. Microsurgical anatomy of the veins of the posterior fossa. J Neurosurg 1983;59:63-105 CrossRef Medline

13. Ardeshiri A, Ardeshiri A, Tonn JC, et al. Microsurgical anatomy of the lateral mesencephalic vein and its meaning for the deep venous outflow of the brain. Neurosurg Rev 2006;29:154-58; discussion 158 Medline

14. Ebner FH, Roser F, Shiozawa T, et al. Petrosal vein occlusion in cerebello-pontine angle tumour surgery: an anatomical study of alternative draining pathways. Eur J Surg Oncol 2009;35:552-56 CrossRef Medline

15. Deda H, Erden I, Yagmurlu B. Evaluation of petrosal sinus patency with 3-dimensional contrast-enhanced magnetic resonance venography in petroclival meningiomas for surgical strategy. Surg Neurol 2005;64(Suppl 2):S67-71 CrossRef Medline

16. $\mathrm{Hu} \mathrm{HH}$, Campeau NG, Huston J 3rd, et al. High-spatial-resolution contrast-enhanced MR angiography of the intracranial venous system with fourfold accelerated two-dimensional sensitivity encoding. Radiology 2007;243:853-61 CrossRef Medline

17. Lee JM, Jung S, Moon KS, et al. Preoperative evaluation of venous systems with 3-dimensional contrast-enhanced magnetic resonance venography in brain tumors: comparison with time-offlight magnetic resonance venography and digital subtraction angiography. Surg Neurol 2005;64:128-33; discussion 133-34 CrossRef Medline

18. Lettau M, Sartor K, Heiland S, et al. 3T high-spatial-resolution contrast-enhanced MR angiography of the intracranial venous system with parallel imaging. AJNR Am J Neuroradiol 2009;30:185-87 CrossRef Medline

19. Nael K, Fenchel M, Salamon N, et al. Three-dimensional cerebral contrast-enhanced magnetic resonance venography at 3.0 Tesla: initial results using highly accelerated parallel acquisition. Invest Radiol 2006;41:763-68 CrossRef Medline

20. Ikushima I, Korogi Y, Kitajima M, et al. Evaluation of drainage patterns of the major anastomotic veins on the lateral surface of the cerebrum using three-dimensional contrast-enhanced MP-RAGE sequence. Eur J Radiol 2006;58:96-101 CrossRef Medline

21. Ardeshiri A, Ardeshiri A, Linn J, et al. Microsurgical anatomy of the mesencephalic veins. J Neurosurg 2007;106:894-99 CrossRef Medline

22. Cohen J. A power primer. Psychol Bull 1992;112:155-59 CrossRef Medline

23. Altman DG. Practical Statistics for Medical Research. Boca Raton: Chapman \& Hall; 1990:624

24. Mizutani K, Toda M, Yoshida K. The analysis of the petrosal vein to prevent venous complications during the anterior transpetrosal approach in the resection of petroclival meningioma. World Neurosurg 2016;93:175-82 CrossRef Medline

25. Hauser TH, Yeon SB, Kissinger KV, et al. Variation in pulmonary vein size during the cardiac cycle: implications for non-electrocardiogram-gated imaging. Am Heart J 2006;152:974.e1-6 Medline 